\title{
The Acoustical Behavior of Contra-Rotating Fan
}

\author{
Juan Wu $\mathbb{D}^{\mathrm{D}}$, Ziming Kou, and Jing Liu \\ School of Mechanical Engineering, Taiyuan University of Technology, Taiyuan, Shanxi 030024, China \\ Correspondence should be addressed to Juan Wu; wujuanz@163.com
}

Received 12 December 2017; Accepted 11 February 2018; Published 1 April 2018

Academic Editor: Sébastien Poncet

Copyright (@) 2018 Juan Wu et al. This is an open access article distributed under the Creative Commons Attribution License, which permits unrestricted use, distribution, and reproduction in any medium, provided the original work is properly cited.

\begin{abstract}
The noise produced by a contra-rotating ventilator can cause injury to humans. Therefore, it is important to reduce noise caused by ventilators. In this study, the Ffowcs Williams and Hawkings (FW-H) model was used to simulate the acoustics of four different axial impeller spacing points based on the unsteady flow field through a FBD No. 8.0 contra-rotating ventilator. Experiments were conducted to verify the correctness of the numerical model. Meanwhile, the Variable Frequency Drive (VFD) drives the two motors of $55 \mathrm{~kW}$ to give the impellers different speeds to distinguish different conditions. The results showed that the main noise source of the ventilator was the two rotating impellers and the area between them. For the same axial space, the noise decreased with the increase of flow rate and then decreased. And the amplitude of the discrete pulse increased gradually. It can be concluded that the vortex acoustics decreased gradually with the increase of flow rate and the rotating acoustics were the major contributor. With the axial distance increasing, the noise caused by the two impellers was weak, and the frequencies of sound pressure level moved toward medium- and low-frequency bands gradually. The suitable axial space could reduce noise and improve the working environment.
\end{abstract}

\section{Introduction}

Contra-rotating (rotation of two rotors in opposite directions) fans were widely used in the ventilation systems of mine and other sites [1]. Contra-rotation fans can realize compact sizes without the need for stators or even higher rotational speeds of one rotor. Because of the large numbers of such devices and their role in mining industry, the noise generated by running contra-rotating fans should be reduced to improve comfortable working environments and human life quality. The installation effects of impellers were important as they can significantly alter the sound radiation as compared to an isolated propeller [2]. Meanwhile, the axial spacing was an important factor which can affect the aerodynamic performance of contra-rotating fan. Therefore, the study of noise generation, propagation, and reduction was one of focuses related to contra-rotating fans.

In the current investigation, both numerical and experimental studies were employed to obtain the characteristics of flow field in contra-rotating fan [3-7]. On axial spacing, Roy and Rao [8] tested flow behavior of contra-rotating fan. The results showed that when the axial gap ranged from $32 \%$ to $110 \%$ of the first fan blade chord, the pressure ratio would decrease by $8 \%$ at most. In addition, the best performance occurred at $49 \%$ axial gap. Zhang et al. [9] found it was meaningless to lengthen the axial gap when it was over $80 \%$ of the chord and the optimal axial gap was about $70 \%$. Shigemitsu et al. [10] discussed the influences on the performance and the noise of axial distance. They showed the interaction between the front and the rear rotors. Akkermans et al. [11] proposed that the differences caused by the aerodynamic performance can be ignored. The most significant factor was interaction tone noise, which can be reduced by the application of trailing edge blowing.

This paper studied the acoustic behavior of FBD No. 8.0 contra-rotating fan. The acoustics of four axial spacing points were simulated by the FW-H noise model based on the unsteady flow field. In addition, experimental measurements of sound pressure levels were collected to validate the numerical simulation results.

\section{Experimental Procedures}

This study was carried out at the contra-rotating test facility at School of Mechanical Engineering, Taiyuan University of Technology. The test system of contra-rotating fan is shown 


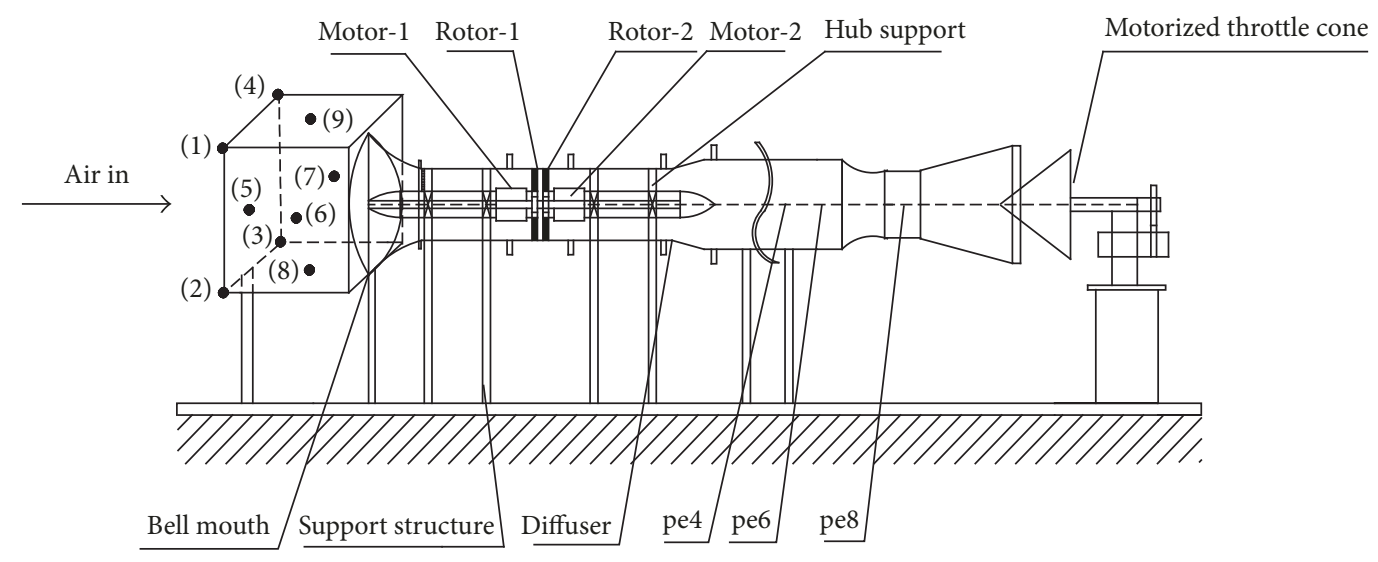

FIGURE 1: Experiment schematic of the contra-rotating fan.

in Figure 1. Two AC motors of $55 \mathrm{~kW}$ with separate Variable Frequency Drive (VFD) were used to independently drive the rotors at different speed combinations [12]. The VFD also had a provision to measure and display the power consumed by the motors, which permits the calculation of the rotor efficiencies. Rotor-1 had 14 blades, and its incidence angle was $46^{\circ}$. Rotor- 2 had 10 blades, and its incidence angle was $30^{\circ}$. The tip-gap between rotor and housing was $2 \mathrm{~mm}$, and both impeller diameters were $800 \mathrm{~mm}$. Their hub ratio was 0.6 and their axial spacing was with a chord of $124 \mathrm{~mm}$. Ducts were attached to the fan's outlet. According to standard GB/T1236-2000 in China, the B-type (pressure-in-type) air duct experimental fan was set up including pressure sensors, hub supports, and the motorized throttle cone, as shown in Figure 1. Various resistances can be got by adjusting the distance between the motorized throttle cone and the test air duct. It was the initial state with no resistance, and the throttle cone was $430 \mathrm{~mm}$ from the outlet of the air duct.

The distance of point pe 4 from the outlet of fan was 5 times the diameter of the fan. The distance of point pe6 from point pe 4 was 3.5 times the diameter of the fan. The distance point pe 8 from point pe 6 was 0.4 times the diameter of the fan. Four monitoring pressure sensors were evenly distributed along the circumferential direction, respectively. The pressure of the fan was obtained by means of the four pressure values of point pe4. And the flow rate could be calculated by Bernoulli principle from the pressure difference $\Delta p$ of point pe6 and point pe8. Finally, taking the environmental parameters into consideration, the test data was calculated to obtain the fan performance in the standard case which was temperature $20^{\circ} \mathrm{C}$, atmospheric pressure $101325 \mathrm{~Pa}$, and air density $1.2 \mathrm{~kg} / \mathrm{m}^{3}$.

The fan noise was measured by using GRAS microphones. A cube was set up at the inlet of fan, and its length was one meter. Meanwhile, this test required the inlet of fan to be on surface of the cube. The typical measurement positions (numbered from (1) to (9)) were distributed around the test fan, shown in Figure 1. The sound intensity was tested by using microphones through scanning method. The uncertainty of the microphones as established by GRAS Sound \& Vibration was $\pm 0.2 \mathrm{~dB}$, with a confidence level of
95\%. The microphone signals were transferred into LMS Test Lab's noise test and analysis module, made by SIEMENS. The data acquisition frequency was $10 \mathrm{kHz}$ per channel.

\section{Simulation Model and Boundary Condition}

In this research, numerical simulation was performed by using FLUENT Ver. 15.0 to investigate the performance and acoustic characteristics of this contra-rotating fan. Fan model and mesh model of the FBD No. 8.0 contra-rotating fan were shown in Figure 2. The flow field of fan was divided into five parts, inlet, rotor-1 area, the area between rotor-1 and rotor-2, rotor- 2 area, and outlet by ICEM software. As the fluidity in the area between rotor- 1 and rotor- 2 was complex, the grid in this area was encrypted to ensure the simulation accuracy. The axial spacing was of 0.5 chord, 0.65 chord, 0.75 chord, and 1 chord, respectively.

Before the numerical simulation, the following assumptions were made as follows: (1) The ambient background was the standard atmospheric pressure. (2) The gas gravity and the buoyancy were neglected. (3) The internal airflow of the fan was turbulent. (4) The turbulence model was used to simulate the flow field. (5) The airflow streamline was continuous during the numerical simulation process in the fan.

The inlet at the bell mouth was used as the simulation inlet combined with a velocity entrance boundary, and the outlet of the diffuser was the simulation outlet combined with a free outflow boundary. First the RNG $k-\varepsilon$ model was used to simulate the steady flow field, and the calculation results were taken as the initial field of unsteady simulation. In steady flow field, the inlet speed was $15 \mathrm{~m} / \mathrm{s}$. Moving reference frame was applied to couple the rotating rotors and stationary casing. The $x$-axis was the rotation axis, setting speed as $2900 \mathrm{rpm}$. The wall boundary condition was nonsliding boundary, and the near wall area used a standard wall function. It was converged when the residuals of the parameters (the velocity in all directions, $k$, and $\varepsilon$ ) were less than $10^{-4}$, and the volume flow rate difference between the inlet and outlet simultaneously reached $10^{-5}$. Numerical method applied in the simulation was summarized in Table 1. 


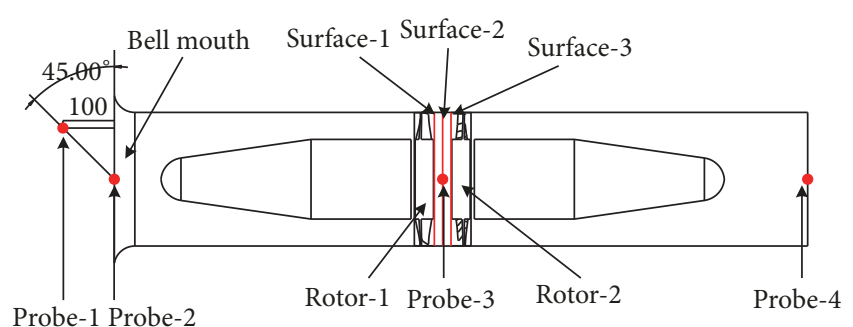

(a) Fan model

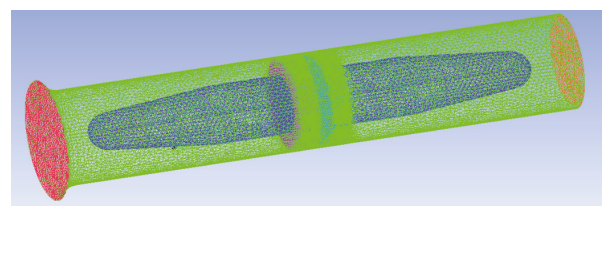

(b) Mesh model

FIGURE 2: Model of FBD No. 8.0 contra-rotating fan.

TABLE 1: Numerical methods of CFD.

\begin{tabular}{lc}
\hline Governing equation & RANS \\
Turbulence model & RNG $k-\varepsilon$ \\
Pressure-velocity coupling & SIMPLEC \\
Pressure solver & Standard \\
Momentum solver & 2nd-order upwind \\
\hline
\end{tabular}

The flow field obtained in the steady simulation was considered as the initial value of unsteady simulation, and the Smagorinsky-Lilly subgrid model was used in unsteady simulation. Considering the accuracy of the calculation and the computer performance, the computational step of the unsteady simulation was set to one hundredth of the rotation speed, which was $0.000206 \mathrm{~s}$, and each time step was 100 times. In addition, the unsteady simulation used second-order implicit time advance scheme and secondorder upwind momentum solver in order to get a more accurate solution. Then, the aeroacoustics under 3D unsteady flow field were simulated by using Large Eddy Simulation (LES). The step of the sound field simulation was set to $0.0000206 \mathrm{~s}$. There were 50 iterations in every time step and the total was 1000 time steps. The sound pressure fluctuations of the monitoring probes are computed by introducing the FW-H model based on the Lighthill sound analogy [13]. The rotating zones of two rotors were selected as the noise source surface. Four noise monitoring probes were randomly arranged in the bell mouth, rotor-1, rotor-2, and diffuser, shown in Figure 2. According to literature, one probe was used in each monitoring zone to obtain the acoustic characteristics in different regions. Fourier transforms were applied to the time domain pressure fluctuation data to obtain the aerodynamic noise spectrum [14].

\section{Simulation Results and Discussion}

The vortices with four axial spacing points of three surfaces are shown in Figure 3. And the three surfaces were surface 1, surface 2, and surface 3, shown in Figure 2. It can be seen that when the surface approaches rotor-2, the total vorticity showed a tendency decrease first and then increase. And the central part vortices of surface were lower than other parts of surface. In addition, with the axial spacing increasing, the vorticity of surface 3 changed obviously. When the axial
TABLE 2: The highest sound pressure of four axial spacing points.

\begin{tabular}{lcccc}
\hline Axial spacing & Probe- 1 & Probe-2 & Probe-3 & Probe-4 \\
\hline 0.5 chord & $92 \mathrm{~dB}$ & $100 \mathrm{~dB}$ & $95 \mathrm{~dB}$ & $100 \mathrm{~dB}$ \\
0.65 chord & $85 \mathrm{~dB}$ & $90 \mathrm{~dB}$ & $95 \mathrm{~dB}$ & $95 \mathrm{~dB}$ \\
0.75 chord & $85 \mathrm{~dB}$ & $95 \mathrm{~dB}$ & $98 \mathrm{~dB}$ & $92 \mathrm{~dB}$ \\
1 chord & $85 \mathrm{~dB}$ & $97 \mathrm{~dB}$ & $98 \mathrm{~dB}$ & $90 \mathrm{~dB}$ \\
\hline
\end{tabular}

spacing was 0.75 chord, the vorticity of surface 3 was more uniform than the others. If the axial spacing was 0.5 chord, the vortex of surface 3 was chaotic. This indicated that too small spacing could hinder smooth flow and lead to deterioration of interstage flow field, although in the design condition. It can be seen that the reduction of the axial spacing caused a more severe eddy current between the two impellers. And the airflow was affected more by two rotors which leads to larger volatility and uneven distribution. For the fans used in mines, 0.5 chord was not the best option. With the axial spacing increasing, impulse between two impellers was weakened, and the vorticity of surface 2 reduced and was uniform. Rotor- 2 produced a high reverse pressure gradient, which had a large effect on the flow field of rotor-1. Although the intervening effect of the two impellers was relatively unavoidable, the suitable spacing was helpful to reduce the vortices.

Figure 4 shows the sound power spectral densities for four axial spacing points at fan entrance (point 2). It can be found that the peak of the sound power spectral density with four axial spacing points was close to $400 \mathrm{~Hz}$ and $1000 \mathrm{~Hz}$ and first decreased and then increased with the axial spacing increasing. These two frequencies were associated with the second harmonic frequency of rotor- 2 and did not coincide with the frequency of rotor-1. The sound power spectral density was more affected by rotor- 2 than rotor-1. In addition, when the axial spacing was 0.5 chord, the sound power spectral density was significantly higher than that for other axial spacing. When the axial spacing was 0.65 chord, the peak of sound power spectral density was lower than others.

Figure 5 shows the sound pressure levels at measuring probes with four axial spacing points. Table 2 shows the highest sound pressure levels at measuring probes. It can be seen from the table that the sound pressure at point $1 \mathrm{did}$ not change significantly with the axial spacing. If the axial spacing was 0.5 chord, the sound pressure of four points 

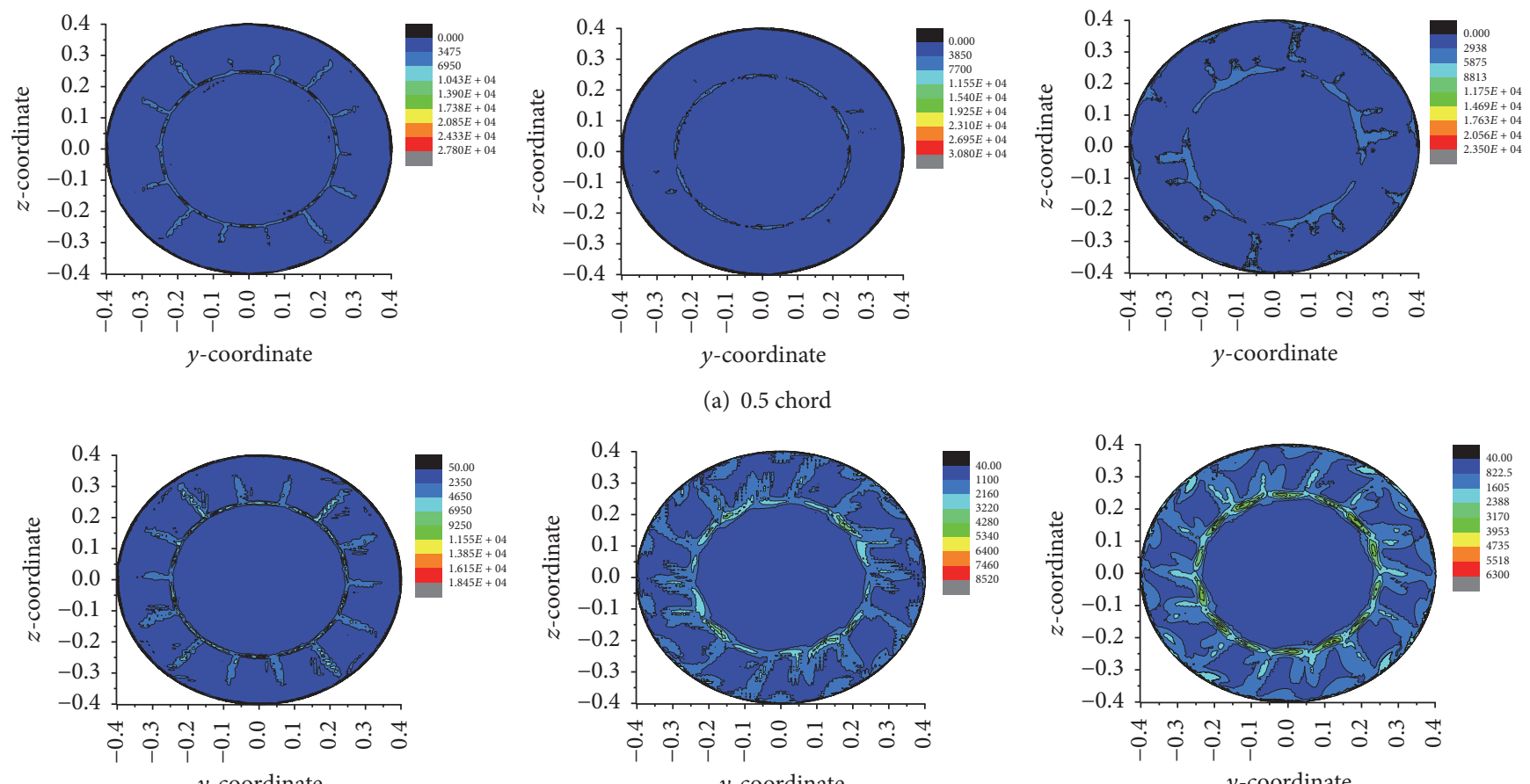

(a) 0.5 chord
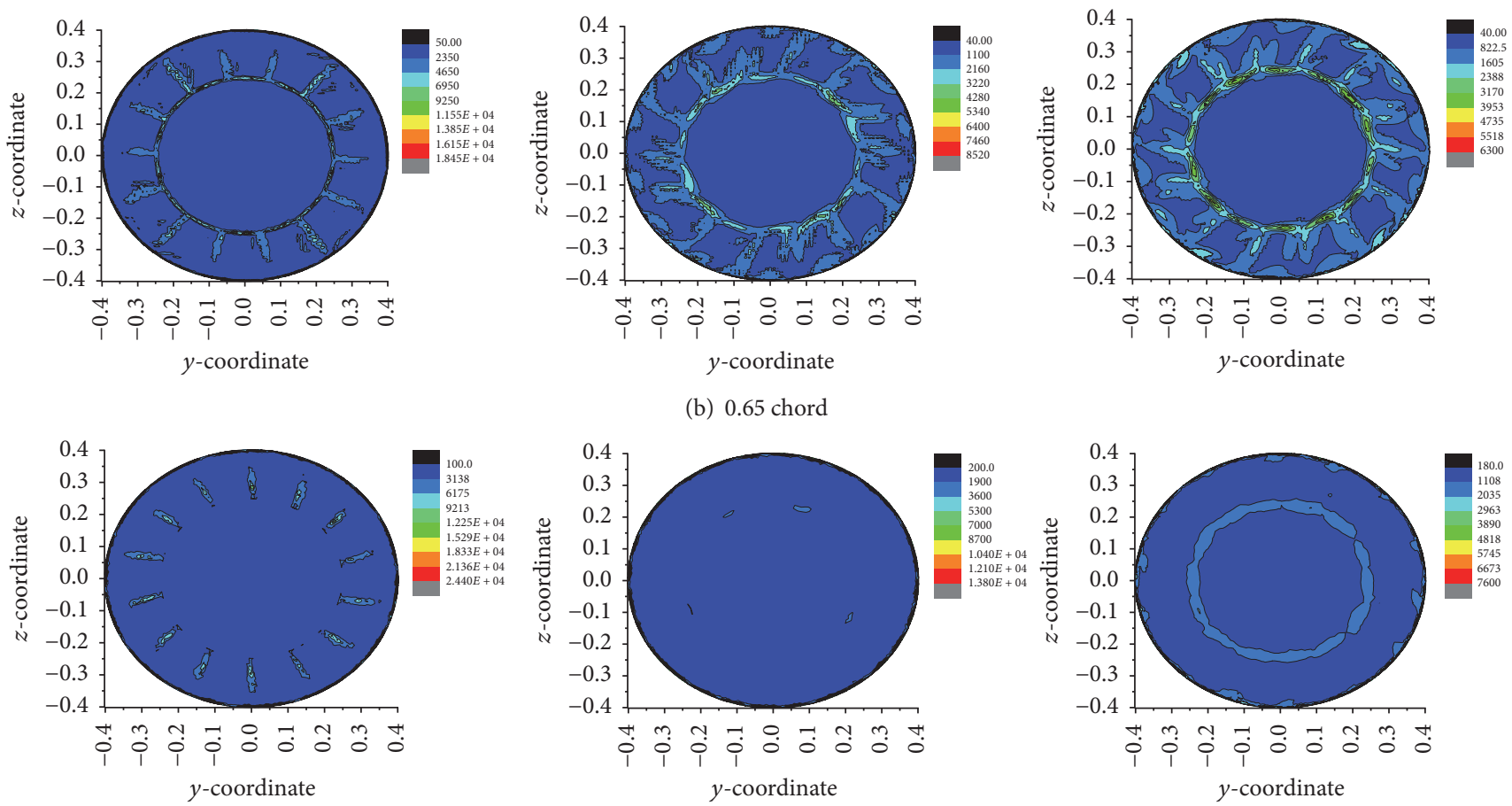

(c) 0.75 chord
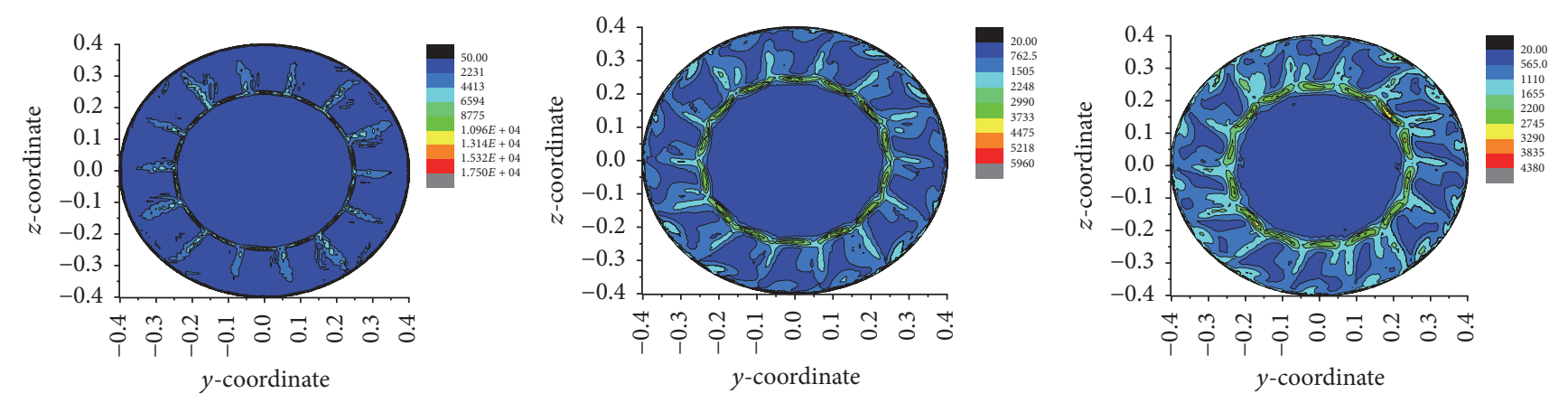

(d) 1 chord

FIGURE 3: The vorticity of three sections under different axial spacing.

was at a high level. The sound pressure at point 2 (the fan inlet) decreased first and then increased with the axial spacing increasing. This was because a strong sound radiation was in the entrance under the smallest axial spacing, which caused a higher sound pressure. When the axial spacing was increasing, the interstage flow field was optimized (shown in
Figure 3). The airflow field tended to be uniform, and the turbulence pulsation in the flow field was weakened; then the maximum sound pressure decreased. But the sound pressure at the outlet of fan was significantly higher than that at the fan inlet. The bell mouth made the flow of air into the fan relatively smooth. Turbulence pulsation phenomenon was 


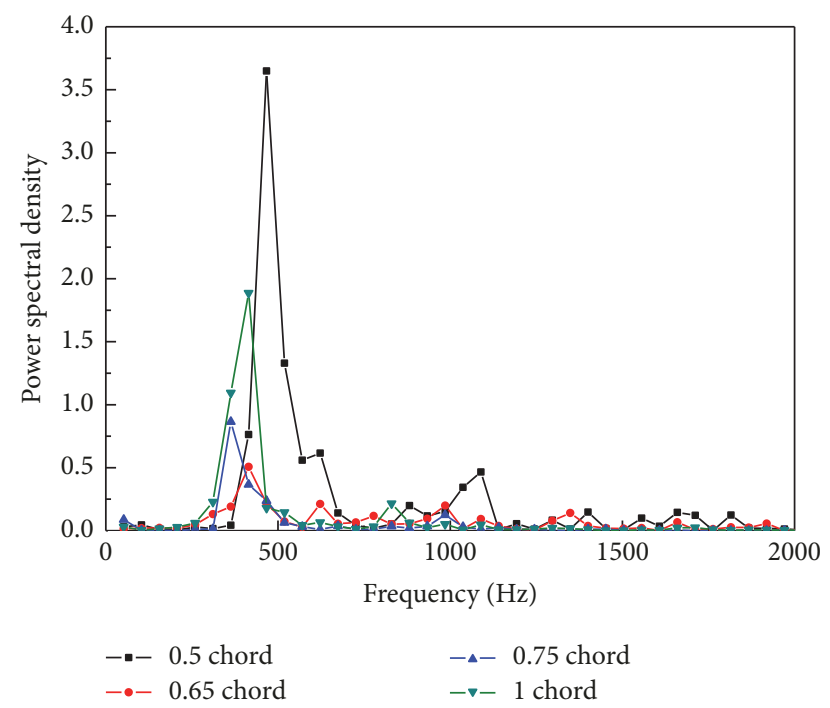

FIGURE 4: The sound power spectral density with four axial spacing points at fan inlet.

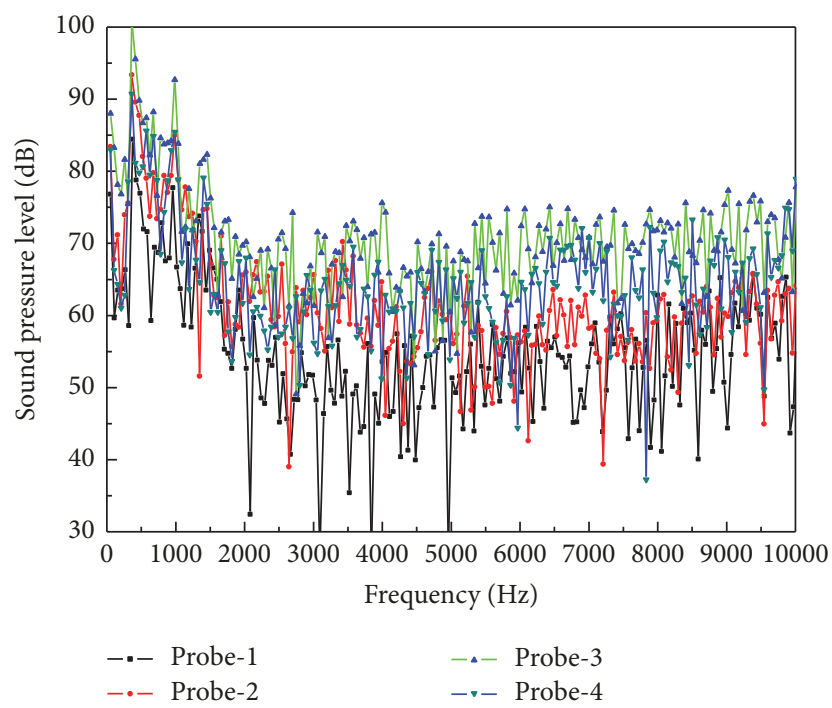

Figure 5: The sound pressure levels at four probes under four axial spacing points.

very small. Through the effect of two rotors, the fan outlet airflow was spiral accompanied by the vortex, which was in accord with Figure 3. So noise at the outlet was greater than the entrance noise.

Figure 6 shows the sound pressure levels at the entrance fan. It can be found that the highest sound pressure appeared near $500 \mathrm{~Hz}$. With the frequency increasing, the sound pressure level decreased first and then was maintained at a certain valve. When the axial spacing was 0.5 chord and 0.65 chord, the sound pressure level declined obviously when frequency was from $500 \mathrm{~Hz}$ to $5000 \mathrm{~Hz}$ and it was relatively higher between $500 \mathrm{~Hz}$ and $4000 \mathrm{~Hz}$, indicating that the fan's noise in these two axial spacing points was mainly broadband noise. When the frequency was higher than $5000 \mathrm{~Hz}$, the sound pressure level was maintained between $50 \mathrm{~dB}$ and

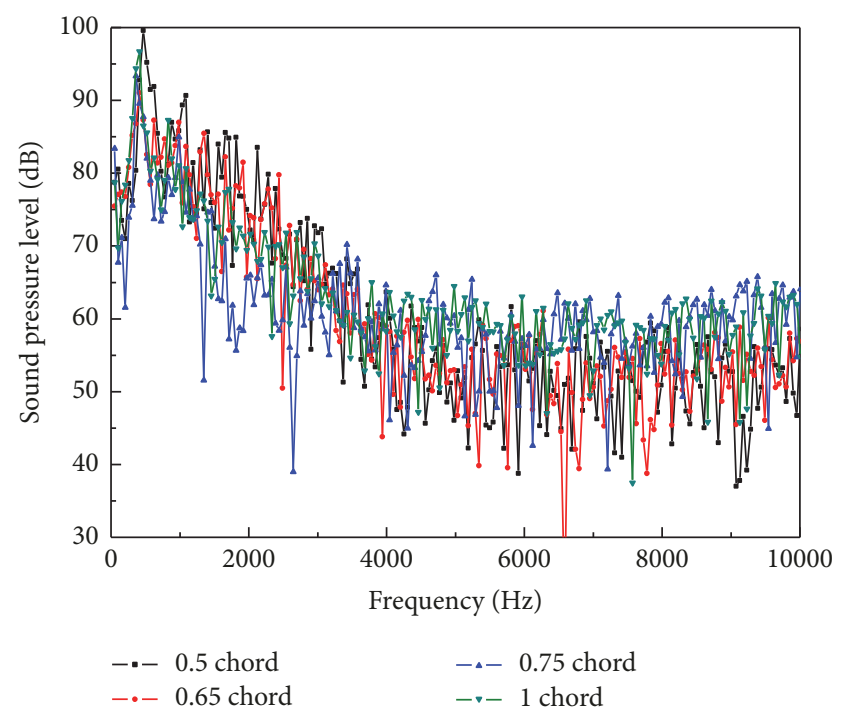

FIgURE 6: The sound pressure levels at the entrance fan.

TABLE 3: The efficiencies of four axial spacing points.

\begin{tabular}{lcccc}
\hline Spacing & 0.5 chord & 0.65 chord & 0.75 chord & 1 chord \\
\hline $\begin{array}{l}\text { The highest } \\
\text { efficiency (\%) }\end{array}$ & 65.1 & 68.59 & 73 & 70.88 \\
\hline
\end{tabular}

$60 \mathrm{~dB}$. But when the axial spacing was 0.75 chord and 1 chord, the sound pressure level was declining from $0 \mathrm{~Hz}$ to $2000 \mathrm{~Hz}$. Away from $2000 \mathrm{~Hz}$, there was little change. With the axial spacing increasing, the frequency of higher sound pressure level changed to lower frequency. According to the numerical simulation results, it can be seen that the large axial spacing was beneficial to weaken the eddy current phenomenon, reduce the generation of eddy current noise, reduce noise, and improve the mining working environment.

It is important to minimize the acoustic noise while maintaining the aerodynamic performance. The aerodynamic efficiencies were based on flow field simulation (shown in Table 3). We can see that when the axial spacing increased, the efficiency increased first and then decreased. Meanwhile, it reached the maximum at 0.75 chord. This was mainly because the small axial spacing made pressure pulsate violently, resulting in a large number of eddy currents and causing a relatively high level of noise. Although the low-frequency noise dropped faster, excessive axial spacing made the flow loss increase significantly. Therefore, in terms of the study, the distance of 0.75 chord can get better overall aerodynamic performance based on acoustic considerations.

\section{Experiment Results and Discussion}

The acoustical behaviors of fan were evaluated by LMS Test Lab's noise test and analysis module. The distance between throttle cone and the outlet of the air duct was $430 \mathrm{~mm}$, $114 \mathrm{~mm}, 65 \mathrm{~mm}, 41 \mathrm{~mm}$, and $28.5 \mathrm{~mm}$, respectively, shown in Table 4 . Three tests were conducted to make sure that the 


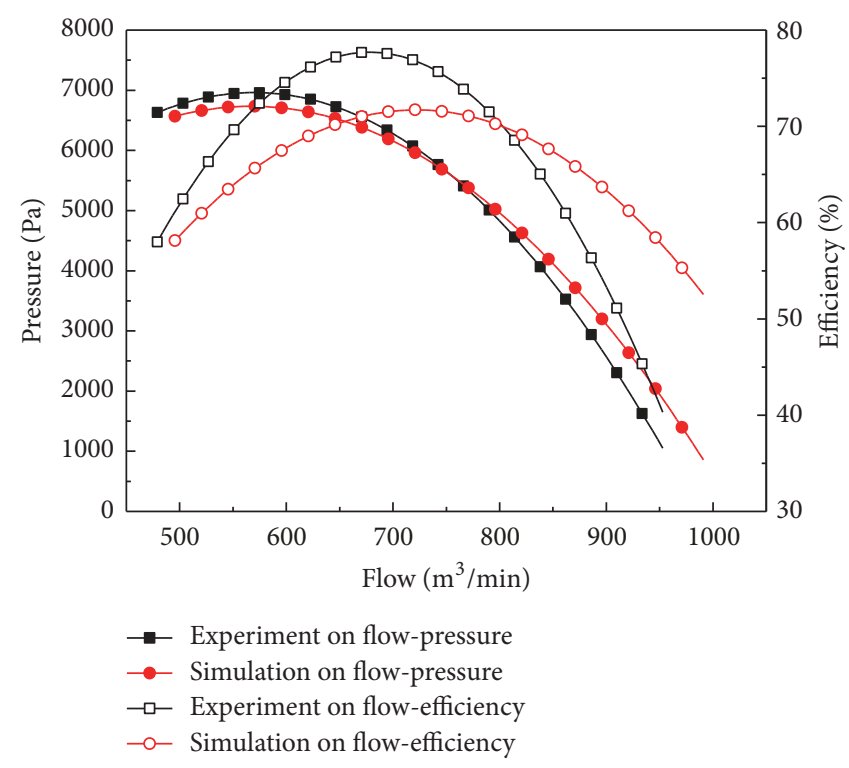

(a) Comparison of flows, pressures, and efficiencies

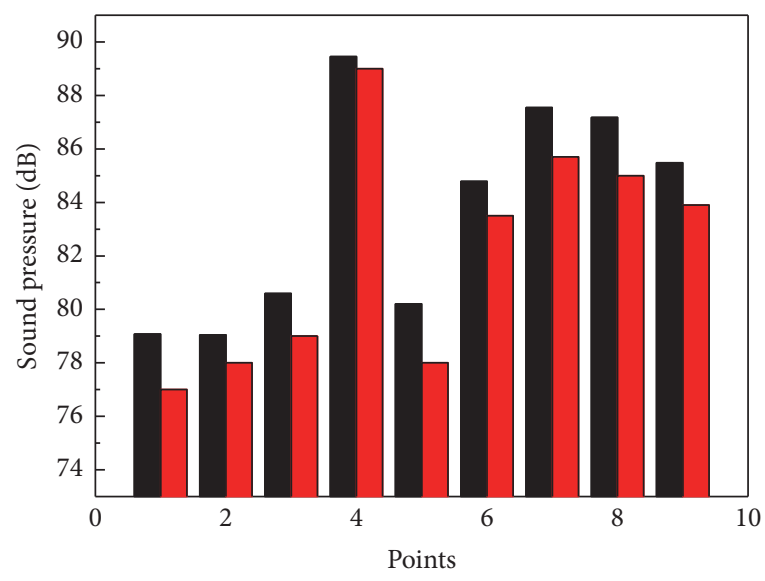

Experiment Simulation

(b) Comparison of sound pressures

FIgURE 7: The comparison of simulation and experiment.

TABLE 4: The test conditions and results.

\begin{tabular}{lccccc}
\hline Number & $\begin{array}{c}\text { Distance } \\
(\mathrm{mm})\end{array}$ & $\begin{array}{c}\text { Speed } \\
(\mathrm{m} / \mathrm{s})\end{array}$ & $\begin{array}{c}\text { Flow } \\
\left(\mathrm{m}^{3} / \mathrm{min}\right)\end{array}$ & $\begin{array}{c}\text { Pressure } \\
(\mathrm{Pa})\end{array}$ & $\begin{array}{c}\text { Efficiency } \\
(\%)\end{array}$ \\
\hline 1 & 430 & 20 & 952.892 & 1120.614 & 33.8 \\
2 & 114 & 17 & 857.454 & 3564.879 & 66 \\
3 & 65 & 15 & 752.752 & 5666.926 & 75.375 \\
4 & 41 & 13 & 639.836 & 6719.235 & 73.475 \\
5 & 28.5 & 10 & 595.033 & 7078.894 & 60.372 \\
\hline
\end{tabular}

repeatability of experimental data is at the same testing condition. The comparison of pressures, flows, and efficiencies between simulation and experiment is shown in Figure 7. The maximum sound pressure level at 9 measurement points (shown in Figure 1) in front of the fan inlet was contrasted with simulation. The efficiency of fan increased first and then decreased when the flow descended. The numerical simulation results were basically in accord with the experimental results, and their deviation on flow rate and pressure was within the allowed range. However, the efficiency results between the numerical simulation and the experiment had a large deviation. This was mainly because physical parameters of the air, the length of fan cylinder, and ductwork model and motor efficiencies were different under numerical simulation method and experiment method. Rotor-1 mainly guaranteed the output of the fan flow, and rotor- 2 was the key to ensure the pressure. Being in no-resistance state, the flow rate was the largest. Rotor-2 shaft power was relatively low; the pressure and efficiency were low too. With the decrease of the flow, the second shaft power increased and the growth rate was faster and the pressure and efficiency increased as well. When the flow reached $15 \mathrm{~m} / \mathrm{s}$ or so, the efficiency reached the highest value which was $75 \%$. When the flow reduced further, the pressure increasing speed slowed down and the efficiency descended. When the flow was less than $10 \mathrm{~m} / \mathrm{s}$ or more, the fan worked unstably and the rotating stall occurred. If the flow rate reduced further, the surge phenomena happened. It was harmful to the fan and should be avoided during the working process.

Comparing the noise spectra of five different conditions (Figure 8), it was not difficult to see that the sound pressure declined obviously within $1000 \mathrm{~Hz}$. When the frequency was above $1000 \mathrm{~Hz}$, the sound pressure was maintained at a level of about $50 \mathrm{~dB}$. If the speed was $20 \mathrm{~m} / \mathrm{s}, 17 \mathrm{~m} / \mathrm{s}$, and $15 \mathrm{~m} / \mathrm{s}$, the discrete peaks of sound pressure emerged at about $50 \mathrm{~Hz}, 500 \mathrm{~Hz}, 700 \mathrm{~Hz}, 1000 \mathrm{~Hz}, 1200 \mathrm{~Hz}$, and $1400 \mathrm{~Hz}$. The fundamental frequency of rotor-1 was $f_{1}=(2900 *$ 14) $/ 60=676 \mathrm{~Hz}$. The fundamental frequency of rotor -2 was $483 \mathrm{~Hz}$. Compared with the basic frequency and the multiplied frequency of two rotors, $500 \mathrm{~Hz}$ and $1000 \mathrm{~Hz}$ were similar to the fundamental frequency and two times frequency of rotor- $2.700 \mathrm{~Hz}$ and $1400 \mathrm{~Hz}$ were matched to the basic frequency and two times frequency of rotor-1. $1200 \mathrm{~Hz}$ was consistent with the frequency of rotary noise $f_{2}=$ $2900 *(14+10) / 60=1160$. The peak of discrete noise in the low-frequency region $(f<500 \mathrm{~Hz})$ decreased too with decreasing the flow speed. There was no discrete peak in the low-frequency region and the rate of sound pressure was 


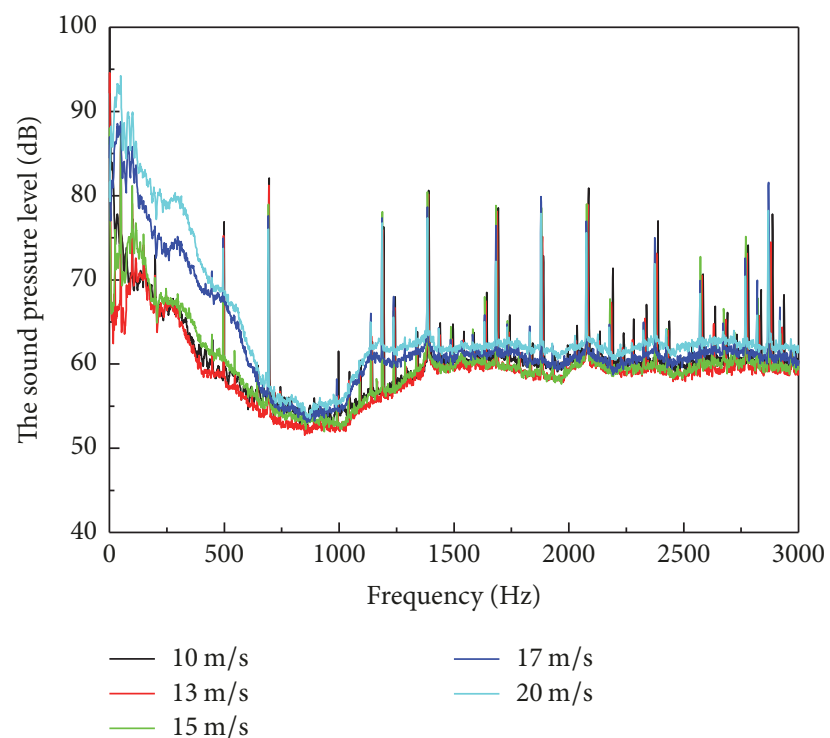

FIGURE 8: Sound pressure test of five different conditions.

inconspicuous when the flow speed was $13 \mathrm{~m} / \mathrm{s}$ and $10 \mathrm{~m} / \mathrm{s}$. The main reason for this phenomenon was that the flow rate of these two speeds was relatively small and there were a large number of vortices in the flow field. And the large number of vortices caused the turbulence noise to increase, indicating that broadband noise was dominant.

\section{Conclusions}

(1) For the special contra-rotating structure, its flow field between rotor-1 and rotor-2 was complicated. The larger axial spacing was beneficial to weaken the vortex phenomena between the two impellers to ensure the uniformity of airflow. But excessive axial spacing would increase the axial size of the fan and affect its applicability. The suitable axial spacing should be chosen to ensure the performance of the fans.

(2) Based on the unsteady simulation of flow field, the FW-H model was applied to simulate the noise spectrum under four axial spacing points. The results showed that the peak frequency of power spectral density was about $500 \mathrm{~Hz}$ and did not change with axial spacing. With frequency ascending, the level of sound pressure of the fan decreased first and then had little change. With axial spacing increasing, the vortex was weakened, and the frequency of sound pressure level gradually moved toward medium- and lowfrequency bands. The contra-rotating fan's noise decreased.

(3) The effect of flow rate on noise spectrum was studied by experiment. The results showed that the peak of discrete noise in the low-frequency region decreased with decreasing the flow speed. When the flow speed was low, broadband noise was dominant.

\section{Conflicts of Interest}

The authors declare that they have no conflicts of interest.

\section{Acknowledgments}

Thanks are due to School of Mechanical Engineering, Taiyuan University of Technology, Taiyuan, Shanxi Province, China, for assistance with the experiments and to Ziming Kou and Jing Liu for valuable discussion. At the same time, the authors acknowledge the support of Shanxi Province Science and Technology Major Projects (MJ2016-2).

\section{References}

[1] Q. G. Chen, W. Sun, F. Li, and Y. J. Zhang, "Air-structure coupling features analysis of mining contra-rotating axial flow fan cascade," in Proceedings of the 6th International Conference on Pumps and Fans with Compressors and Wind Turbines, ICPF 2013, China, September 2013.

[2] R. A. D. Akkermans, M. Pott-Pollenske, H. Buchholz, J. W. Delfs, and D. Almoneit, "Installation effects of a propeller mounted on a high-lift wing with a coanda flap. part I: Aeroacoustic experiments," in Proceedings of the 20th AIAA/CEAS Aeroacoustics Conference 2014, usa, June 2014.

[3] I. A. Brailko, V. I. Mileshin, A. M. Volkov, and V. N. Korznev, "Numerical and experimental investigations of crf with simulation of flow nonuniformity in the basic flight conditions," in Proceedings of the 27th Congress of the International Council of the Aeronautical Sciences 2010, ICAS 2010, pp. 2619-2627, fra, September 2010.

[4] B. Roy, H. kumar, and A. Batra, "Effect of inlet distortions, coswirl and counter-swirl on single axial fan and contra-rotating axial fans," in Proceedings of ISABE, 2001.

[5] C. S. Mistry and A. M. Pradeep, "Effect of variation in axial spacing and rotor speed combinations on the performance of a high aspect ratio contra-rotating axial fan stage," Proceedings of the Institution of Mechanical Engineers, Part A: Journal of Power and Energy, vol. 227, no. 2, pp. 138-146, 2013.

[6] C. Mistry and A. M. Pradeep, "Influence of circumferential inflow distortion on the performance of a low speed, high aspect ratio contra rotating axial fan," Journal of Turbomachinery, vol. 136, no. 7, Article ID 071009, 2014.

[7] C. S. Mistry and A. M. Pradeep, "Study of the velocity flow field under distorted inflow conditions for a high aspect ratio low speed contra rotating fan," in Proceedings of the ASME 2013 Gas Turbine India Conference, GTINDIA 2013, ind, December 2013.

[8] B. Roy and P. S. Rao, "Ducted contra-rotating axial flow fan performance studies," American Society of Mechanical Engineers, vol. 4, pp. 469-474, 1989.

[9] Y. J. Zhang, X. Xu, and Q. G. Chen, "Effects of axial gap on contra-rotating mine fan's performance," Meitan Xuebao, vol. 36, pp. 1217-1221, 2011.

[10] T. Shigemitsu, J. Fukutomi, and H. Shimizu, "Influence of blade row distance on performance and flow condition of contrarotating small-sized axial fan," The International Journal of Fluid Machinery and Systems, vol. 5, no. 4, pp. 161-167, 2012.

[11] R. A. D. Akkermans, A. Stuermer, and J. W. Delfs, "Active flow control for interaction noise reduction of contra-rotating open rotors," AIAA Journal, vol. 54, no. 4, pp. 1413-1423, 2016.

[12] R. A. D. Akkermans, J. W. Delfs, M. Lummer, M. Siefert, B. Caruelle, and C. Tiedemann, "Handling of non-periodic contra rotating open rotor data," in Proceedings of the 18th AIAA/CEAS Aeroacoustics Conference 2012 (33rd AIAA Aeroacoustics Conference), usa, June 2012. 
[13] J. E. F. Williams and D. L. Hawkings, "Sound generation by turbulence and surfaces in arbitrary motion," Philosophical Transactions of the Royal Society A: Mathematical, Physical \& Engineering Sciences, vol. 264, no. 1151, pp. 321-342, 1969.

[14] C. Li, Q. Lin, X. Ding, and X. Ye, "Performance, aeroacoustics and feature extraction of an axial flow fan with abnormal blade angle," Energy, vol. 103, pp. 322-339, 2016. 


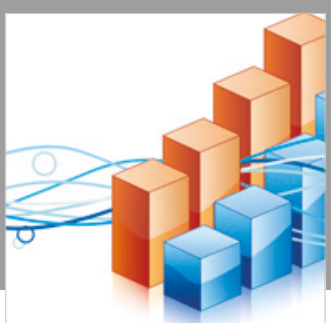

Advances in

Operations Research

\section{-n-m}
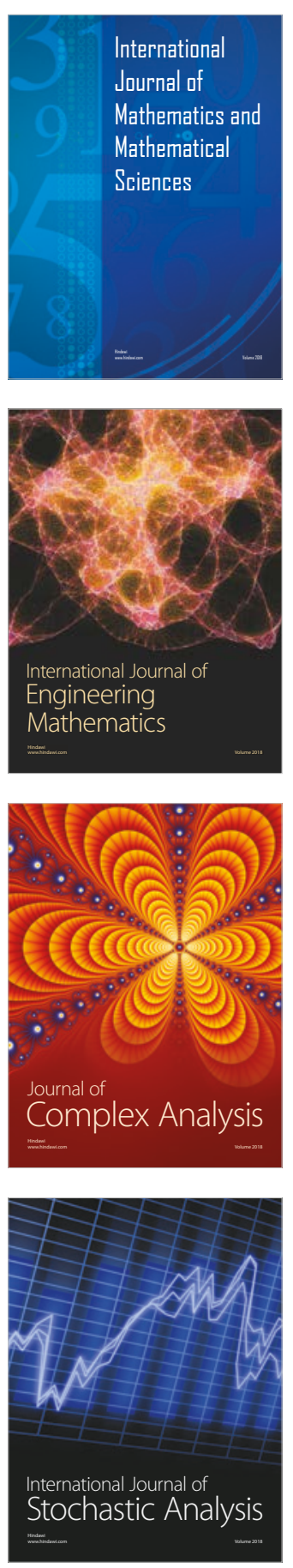
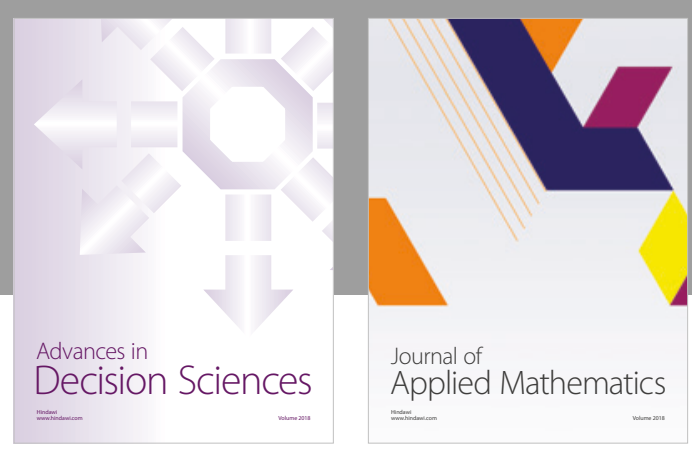

Journal of

Applied Mathematics
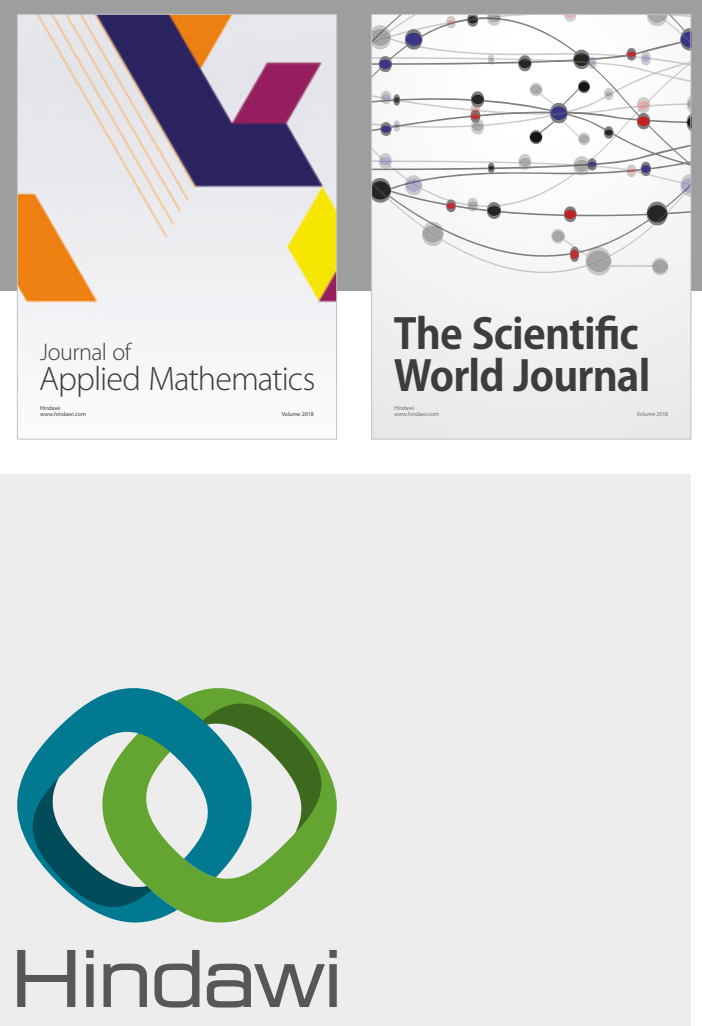

Submit your manuscripts at

www.hindawi.com

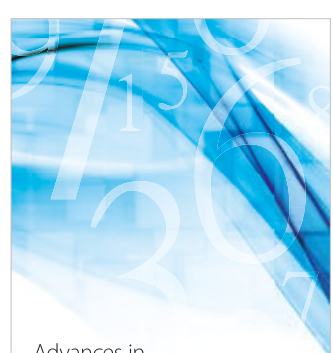

Advances in
Numerical Analysis
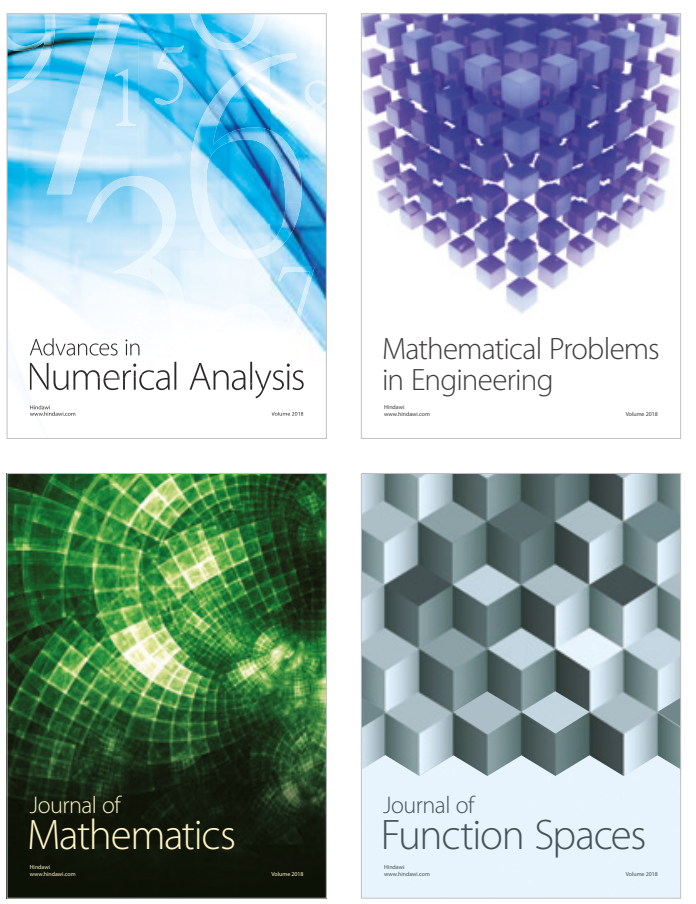

Mathematical Problems in Engineering

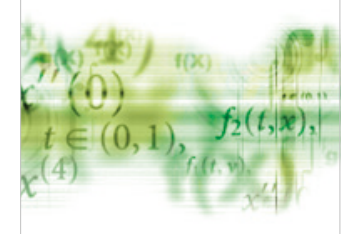

International Journal of

Differential Equations

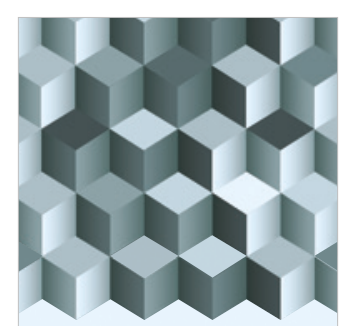

Journal of

Function Spaces

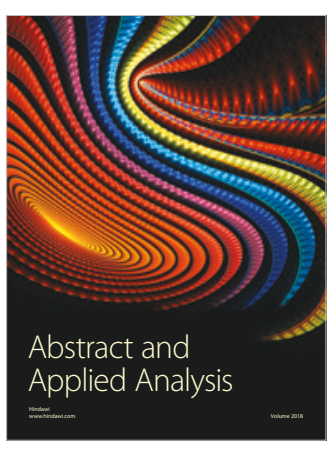

The Scientific

World Journal

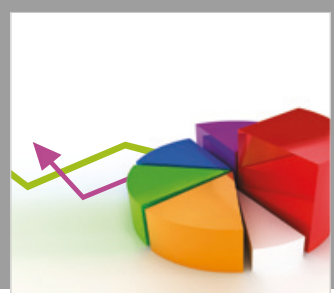

Journal of

Probability and Statistics
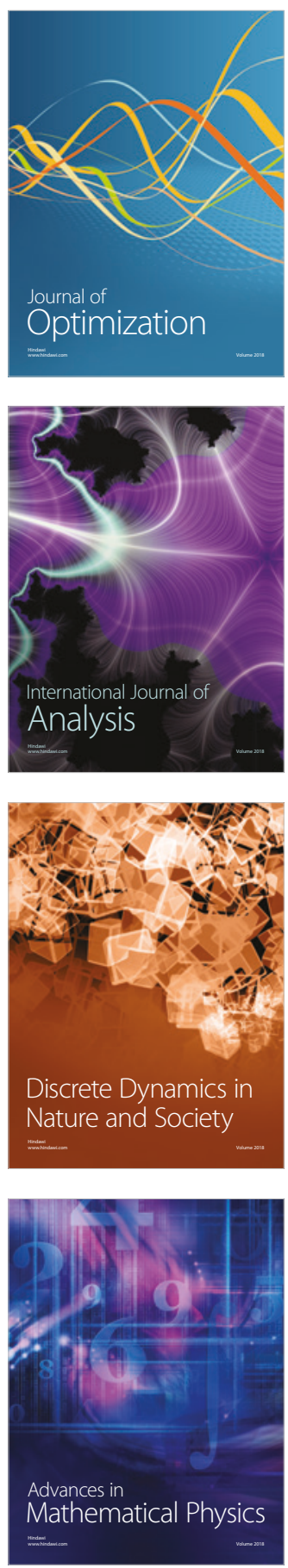